\title{
Sostenibilidad de la estrategia Municipios Saludables en Guatemala
}

\author{
Jorge Laureano-Eugenio ${ }^{1}$, Raúl Otoniel Gómez-Rodríguez², Jhunny Tasejo-Corzantes ${ }^{2}$, Augusto \\ Silvestre Ramírez ${ }^{2}$, Rosa María Pretell Aguilar ${ }^{3}$ y Jacqueline Elizabeth Alcalde-Rabana/4
}

Forma de citar

\begin{abstract}
Laureano-Eugenio J, Gómez-Rodríguez RO, Tasejo-Corzantes J, Silvestre-Ramírez A, Pretell Aguilar RM, Alcalde-Rabanal JE. Sostenibilidad de la estrategia Municipios Saludables en Guatemala. Rev Panam Salud Publica. 2021;45:e37. https://doi.org/10.26633/RPSP.2021.37
\end{abstract}

RESUMEN

Objetivo. Evaluar la sostenibilidad de la estrategia Municipios Saludables en Guatemala, para disponer de evidencia sólida que permita apoyar la toma de decisiones.

Métodos. Se realizó un estudio mixto concurrente en cinco fases: 1) teórica-conceptual, se realizó una revisión narrativa de literatura en sostenibilidad, con base en la cual se propusieron dimensiones y categorías para su evaluación; 2) empírica, se seleccionaron por conveniencia cuatro municipios y se realizaron 29 entrevistas semiestructuradas y cuatro grupos focales a actores clave para explorar la sostenibilidad; con esta información, se asignó un puntaje a cada categoría y dimensión; 3) analítica, por categoría y dimensión, se realizó el análisis del contenido para la información cualitativa y sumatorias y promedios para la información cuantitativa; 4) integrativa, los datos cualitativos se integraron en matrices por categoría y dimensión; y los datos cuantitativos se respaldaron con información cualitativa; y 5) metainferencia, se reflexionó sobre el contexto y su influencia en los resultados.

Resultados. Participaron 92 informantes. En la dimensión operativa, se destacan avances en la transferencia y el uso de resultados, así como en el relevo de liderazgo. En la dimensión legal y política, se destacaron la rendición de cuentas y los planes locales. En la dimensión económica, se destacó la inversión progresiva en salud, agua y saneamiento, con deficiencia de inversiones en determinantes sociales de la salud. En la dimensión social, se observaron pocos mecanismos para impulsar y fortalecer la participación social.

Conclusiones. En los municipios que participaron del estudio se observó un nivel regular de sostenibilidad de la estrategia Municipios Saludables.

Palabras clave Estudio de evaluación; ciudad saludable; promoción de la salud; gobierno local; Guatemala.

La iniciativa Municipios Saludables es impulsada por la Organización Panamericana de la Salud y la Organización Mundial de la Salud (OMS). Esta iniciativa se fundamenta en los principios de atención primaria en salud presentados en Alma-Ata en 1978, así como en la Carta de Ottawa, elaborada durante la Primera Conferencia Internacional de Promoción de la Salud en 1986, en la que se insta a fomentar la creación de ambientes saludables (1). La estrategia Municipios Saludables (EMS) busca posicionar la promoción de la salud (PS) como centro de acción y como prioridad de la agenda política local en salud. Se sustenta en la acción intersectorial, la participación comunitaria y la interculturalidad (2), por lo que busca la participación de autoridades locales gubernamentales, la comunidad y otros actores (3).

Un municipio saludable es aquel en el que "las autoridades, instituciones y ciudadanos trabajan conjuntamente a favor de

\footnotetext{
Secretaría de Salud de Jalisco, Jalisco, México.

Ministerio de Salud y Asistencia Social, Ciudad de Guatemala, Guatemala.

Ministerio de Salud, Lima, Perú.
} 4 Instituto Nacional de Salud Pública, Morelos, México. $\square$ Jacqueline Elizabeth
Alcalde-Rabanal, jackeline.alcalde@gmail.com 
la salud y el bienestar de sus habitantes, abordando los determinantes de la salud que son responsabilidad de los gobiernos y sociedad civil" (4). En 1992, en la Conferencia Internacional de Promoción de la Salud y Equidad, Guatemala asumió el compromiso de fomentar ambientes y entornos saludables (5). En 1999, encargó su implementación al Departamento de Promoción de la Salud, para lo cual se conformó en las provincias una comisión integrada por la municipalidad, el sector salud y otras organizaciones locales. También se formaron comisiones comunitarias para fomentar la participación social y promover políticas de salud a nivel local. Como parte de su aplicación, se realizó un diagnóstico y se oficializó el Plan Municipal de Salud para incidir sobre los determinantes sociales y ambientales $(6,7)$.

Estas iniciativas ponen en evidencia el esfuerzo desplegado por el Ministerio de Salud Pública y Asistencia Social, las municipalidades y las organizaciones comunitarias para implementar la EMS durante más de dos décadas en Guatemala. Esta temporalidad de su implementación hace necesario preguntarse cuál es el nivel de sostenibilidad de la EMS. La respuesta a esta pregunta empieza por definir la sostenibilidad. En teoría, el término sostenibilidad hace referencia a una acción que se puede sostener; es decir capaz de mantenerse durante un tiempo considerado largo (8). Bajo esta concepción, la sostenibilidad de la EMS se concibe como la capacidad de los actores involucrados para mantener de forma continua las actividades y resultados de la estrategia. Para esto, se requiere desarrollar procesos que respondan a las características del contexto social, cultural, político y económico en el que se lleva a cabo una intervención. Una intervención sostenible busca la mejora continua de una situación (9), y la EMS busca contribuir a mejorar de forma constante las condiciones de salud de la población (10).

La implementación de una intervención requiere de recursos financieros y de la vinculación entre actores gubernamentales y la sociedad civil, así como tener en cuenta las consideraciones jurídico-institucionales que faciliten su implementación (11). Por este motivo, para que una intervención tenga resultados sostenibles, se requiere de procesos que se realicen de manera continua en el tiempo (9). En consecuencia, se debe analizar la sostenibilidad desde una perspectiva holística; una de las variables principales es la permanencia en el tiempo; la otra, las dimensiones (12) de su implementación (operativa, políticolegal, económica y social). El estudio de la dimensión operativa se orienta a analizar la capacidad técnica institucional (liderazgo, capacitación de recursos humanos y capacidad de gestión) y operativa (disponibilidad de recursos humanos, insumos y materiales) (13). La dimensión político-legal analiza la existencia de acuerdos claros y mensurables, fijados previamente, que facilitan u obstaculizan la implementación (9). La dimensión económica analiza la disponibilidad e inversión económica para la implementación de una intervención en el mediano y largo plazo (14). Por último, la dimensión social analiza el involucramiento activo y crítico de la sociedad en las intervenciones, así como la legitimidad para demandar y proponer acciones para la continuidad de la estrategia (15).

No se encontraron estudios previos que den cuenta de la sostenibilidad de la EMS. Es así que el presente estudio es relevante, ya que por primera vez se analiza la implementación de la EMS desde la perspectiva de la sostenibilidad. El artículo tiene como objetivo evaluar la sostenibilidad de la estrategia Municipios Saludables en Guatemala, para disponer de evidencia sólida que permita apoyar la toma de decisiones al Ministerio de Salud y Asistencia Social e instancias involucradas a fin de fortalecer la sostenibilidad de sus resultados.

\section{MATERIALES Y MÉTODOS}

Se realizó una evaluación de la sostenibilidad de la EMS en Guatemala para el período 2015-2019 con un diseño mixto (cualitativo y cuantitativo) concurrente (16). A continuación, se describen las fases del estudio (figura 1):

1. Fase teórica-conceptual. Ante la ausencia de estudios previos que evaluaran la sostenibilidad de intervenciones, la tarea inicial fue identificar las dimensiones para evaluarla. Para ello, se realizó una revisión narrativa de literatura disponible sobre sostenibilidad en PS. La búsqueda contempló publicaciones de los cinco últimos años para América Latina y el Caribe y se realizó en PubMed, LILACS y Google Académico. Los términos de búsqueda en español fueron "sostenibilidad", "continuidad de estrategias" y "seguimiento de estrategias", y las palabras en inglés fueron sustainability, strategy continuity y strategy monitoring. Estas se utilizaron con operadores booleanos $(\mathrm{Y}, \mathrm{O})$ más la frase "promoción de la salud" o Health Promotion. Se encontraron 312 artículos, de los cuales se revisó el resumen; 36 artículos conceptualizaron o midieron la sostenibilidad y pasaron a revisión en profundidad; 22 de ellos desarrollaron dimensiones y categorías para evaluar la sostenibilidad.

Se identificaron cuatro dimensiones y 22 categorías para la evaluación. Esta propuesta fue compartida y discutida en modo virtual con siete expertos con formación y amplia experiencia en PS de Chile, Guatemala, México y Perú (dos directores de PS, un investigador en PS, dos asesores en la implementación de la EMS y un coordinador nacional de PS), que consideraron cuatro dimensiones y 18 categorías para evaluar la sostenibilidad (cuadro 1).

2. Fase empírica. Se seleccionaron a conveniencia cuatro 4 municipios según los siguientes criterios: a) haber implementado la EMS desde el año 2000; b) haber recibido apoyo técnico y financiero de la OMS y el Ministerio de Salud; c) haber implementado proyectos con recursos locales; d) ocupar los primeros lugares del ranking de la EMS entre 2015-2019; y e) tener población rural-urbana e indígena-no indígena. A cada municipio se le asignó una letra (A, B , C y D) para mantener el anonimato de la información.

Se realizaron 29 entrevistas semiestructuradas a actores involucrados en la implementación de la EMS de los niveles nacional y municipal, para lo cual se utilizó una guía que exploró las dimensiones operativas, político-legales, financieras y sociales de la sostenibilidad. El muestreo se realizó utilizando la bola de nieve y se detuvieron las entrevistas cuando dejó de aparecer información nueva en las categorías. En el nivel nacional, se entrevistaron coordinadores de instituciones de gobierno encargados de la EMS. A nivel municipal, se entrevistaron actores que implementaron la estrategia (alcalde, regidores o delegados), representantes de otras instituciones (coordinadores de programas de gobierno, organizaciones de la sociedad civil o instancias internacionales) y población (comités comunitarios de salud, comités de desarrollo comunitario y líderes sociales). 
FIGURA 1. Fases del estudio

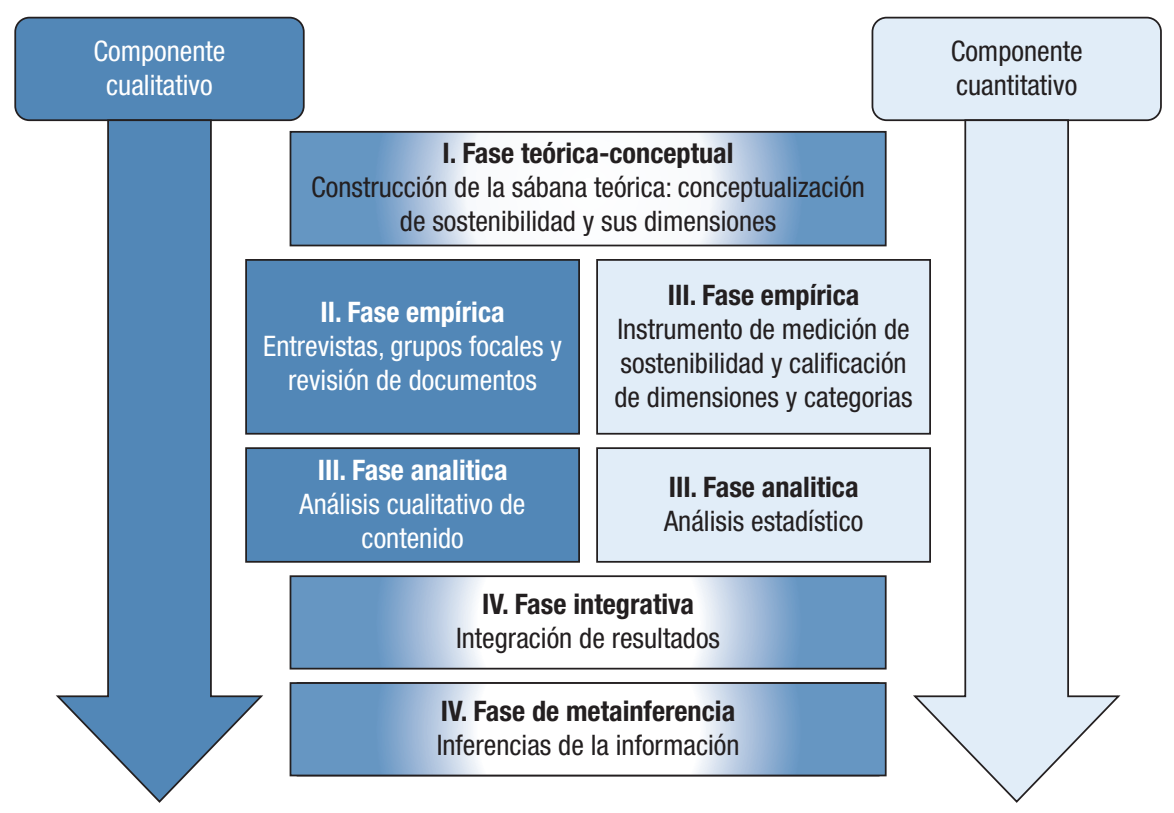

Fuente: elaboración propia de los autores.

CUADRO 1. Categorías y dimensiones para el estudio de sostenibilidad en promoción de la salud

\begin{tabular}{|c|c|}
\hline Dimensiones & Categorías \\
\hline 1. Sostenibilidad operativa & $\begin{array}{l}\text { 1.1 Disponibilidad del personal } \\
\text { 1.2 Transferencia y uso de resultados } \\
\text { 1.3 Acción intersectorial } \\
\text { 1.4 Relevo de liderazgo } \\
\text { 1.5 Monitoreo y seguimiento } \\
\text { 1.6 Evaluación de la estrategia }\end{array}$ \\
\hline 2. Sostenibilidad político-legal & $\begin{array}{l}\text { 2.1 Acuerdos y convenios legales locales } \\
\text { 2.2 Planes locales con enfoque de desarrollo sostenible y promoción de la salud } \\
\text { 2.3 Lineamientos para la operación de la estrategia } \\
\text { 2.4 Rendición de cuentas }\end{array}$ \\
\hline 3.Sostenibilidad económica & $\begin{array}{l}\text { 3.1 Recursos económicos para salud, agua y saneamiento } \\
\text { 3.2 Recursos económicos para acciones operativas } \\
\text { 3.2 Recursos económicos para evaluación }\end{array}$ \\
\hline 4. Sostenibilidad social & $\begin{array}{l}\text { 4.1 Involucramiento activo de la población } \\
\text { 4.2 Aceptabilidad de la estrategia } \\
\text { 4.3 Autonomía y capacidad propositiva de la población } \\
4.4 \text { Utilización de tecnologías de innovación e interacción social } \\
\text { 4.5 Nivel de incidencia }\end{array}$ \\
\hline
\end{tabular}

Fuente: elaboración propia de los autores.

Se realizaron cuatro grupos focales (uno en cada municipio) para explorar las dimensiones de la sostenibilidad. En cada grupo focal participaron entre nueve y 16 informantes (coordinadores de instituciones de gobierno, organizaciones de la sociedad civil, organizaciones internacionales, comités comunitarios de salud, comités de desarrollo comunitario y líderes sociales). Las entrevistas y grupos focales se realizaron en horarios acordados con los informantes y en espacios que aseguraron la privacidad. Antes de su realización, los informantes firmaron un consentimiento. Las entrevistas y grupos focales fueron grabados y trascritos a un procesador de textos. Además, se revisaron informes de la EMS, planes e informes de evaluación.
Para la calificación cuantitativa de cada dimensión de la sostenibilidad en cada municipio, en primer lugar, se calificaron las categorías. A cada categoría se le asignó un puntaje de 0 a $5(0=$ nulo, $1=$ malo, $2=$ regular, $3=$ bueno y $4=$ muy bueno), según su nivel de avance. Con la finalidad de consensuar la calificación para cada categoría, los investigadores elaboraron, con apoyo de los expertos, un instrumento en el que describieron los criterios para calificar cada valor.

3. Fase analítica. En esta fase se realizaron la calificación cualitativa y cuantitativa:

a. Cualitativa. Se transcribieron las entrevistas y grupos focales y se realizó el análisis de contenido según dimensiones y categorías (cuadro 1). Para la 
CUADRO 2. Participantes en entrevistas y grupos focales de la estrategia Municipios Saludables en Guatemala, 2019

\begin{tabular}{|c|c|c|c|c|c|c|c|}
\hline Municipio & Alcalde & Funcionario municipal & $\begin{array}{c}\text { Funcionario } \\
\text { MSPAS }^{b} \\
\text { municipal }\end{array}$ & $\begin{array}{l}\text { Funcionario } \\
\text { MSPAS }^{b} \\
\text { departamental }\end{array}$ & $\begin{array}{l}\text { Funcionario } \\
\text { nacional }\end{array}$ & $\begin{array}{l}\text { Otros organismos } \\
\text { y sociedad civilc }\end{array}$ & Total \\
\hline$A$ & 1 & 5 & 2 & 2 & & 20 & 30 \\
\hline$B$ & & 3 & 2 & 2 & & 11 & 18 \\
\hline C & 1 & 4 & 2 & 2 & & 10 & 19 \\
\hline$D$ & 1 & 4 & 2 & 2 & & 11 & 20 \\
\hline $\mathrm{E}^{\mathrm{a}}$ & & & & & 5 & & 5 \\
\hline Total & 3 & 16 & 8 & 8 & 5 & 52 & 92 \\
\hline
\end{tabular}

codificación se utilizó el software NVivo Versión 12 Plus $^{\circledR}$.

b. Cuantitativa. A partir de la calificación asignada, se obtuvo el puntaje para cada categoría en cada municipio, así como la calificación promedio para los cuatro municipios.

4. Fase integrativa. Se organizaron matrices con información por dimensión y categoría para cada municipio a partir de información procedente de entrevistas, grupos focales y documentos. Con esta información, los investigadores calificaron, de forma individual, cada categoría para cada municipio. Esta calificación fue revisada y discutida con el grupo de expertos y, por consenso, asignaron una calificación final. El puntaje para cada dimensión se obtuvo del promedio de puntajes de sus categorías. Este resultado se contrastó con los informes de las matrices y se compartió con los informantes para validar el puntaje asignado a cada categoría y dimensión. Luego, se graficaron los resultados, a los cuales se integró la información cualitativa.

5. Fase de metainferencia. Los investigadores reflexionaron sobre los hallazgos cuantitativos y cualitativos de la sostenibilidad de manera conjunta. Se analizó el contexto político, social y cultural, así como los referentes históricos, normativos, legales e institucionales de PS en Guatemala en los últimos cinco años para buscar respuestas a los resultados alcanzados y su variabilidad en los municipios.

El presente estudio obtuvo la aprobación del Comité de Ética del Ministerio de Salud Pública y Asistencia Social de Guatemala y del Comité de Ética de la Organización Panamericana de la Salud (PAHOERC).

\section{RESULTADOS}

Participaron 92 actores en las entrevistas y grupos focales, 44 (48\%) fueron hombres y 48 (52\%) mujeres, con edades de 19 a 66 años. La mayoría tenían estudios de secundaria y su permanencia en la implementación de la EMS osciló entre uno y seis años. La mayoría pertenecieron a organizaciones de la sociedad civil (cuadro 2).

\section{Sostenibilidad operativa}

En la evaluación de la dimensión operativa de la sostenibilidad (figura 2a) se observaron avances diferenciados por municipio. Los avances más importantes se identificaron en la transferencia y el uso de los resultados y en el relevo de liderazgo; estas categorías alcanzaron puntajes de 3 y 2,5; respectivamente. En el municipio A se observó mayor disponibilidad del personal para la implementación de la estrategia, en el municipio D, esta disponibilidad fue nula. Los municipios A y B incluyeron personal para PS en su estructura organizacional mientras que los municipios C y D no lo incluyeron, y sólo consideraron representantes o comisiones municipales de salud.

En la transferencia y el uso de resultados, el municipio C presenta el mayor avance, y el municipio D, el menor avance. En la acción intersectorial y el relevo de liderazgo, todos los municipios evidencian el mismo desempeño, con puntajes de 2 a 2,5. La acción intersectorial en los municipios A y B se realiza entre el Gobierno Municipal y Ministerio de Salud; y en los municipios C y D se suman organismos de cooperación internacional.

En tres de cuatro municipios se observó que, ante el cambio de gobierno local, las actividades de PS se mantienen. Sin embargo, en el municipio D, las nuevas autoridades no continúan con actividades asumidas por el gobierno anterior.

"...Por cuestiones evidentemente políticas, el municipio ha sufrido bastante, como no pertenece al partido del gobierno central, se dificulta que continue con sus actividades". DPM-C

En cuanto al monitoreo, el seguimiento y la evaluación, todos los municipios tienen problemas para su ejecución. Los cuatro municipios realizan evaluación básicamente financiera y reconocen que no tienen personal capacitado para la evaluación, por lo que, en estos casos, solicitan apoyo a otras instancias.

\section{Sostenibilidad legal y política}

En la figura $2 \mathrm{~b}$ se observa que la categoría con mayor avance es la rendición de cuentas, en promedio alcanzó un puntaje de 3; la existencia de planes locales alcanzó 2,7; y la existencia de convenios locales, 2,5. El municipio con mayor desarrollo en sostenibilidad legal y política es el municipio $C$, y el que tiene menos avances es el D. Como parte de la rendición de cuentas, los municipios publican memorias de sus logros trimestrales y lo comparten con el Consejo de Desarrollo Municipal. Los planes y acuerdos locales consideraron proyectos para incidir en los determinantes de la salud. 
FIGURA 2. Dimensiones de la sostenibilidad. A, dimensión operativa; B, dimensión político-legal; C, dimensión económica; D, dimensión social


"...nos inculcaron ideas para mejorar la vida, como ordenar y organizar las viviendas, lavado de manos, alimentación sana para los niños. Si un niño crece desnutrido no tiene posibilidades futuras". AM-D.

En regulación, se destacan avances en agua y saneamiento, ordenamiento territorial y construcción, entre otros. Ningún municipio cuenta con lineamientos operativos para la EMS y el nivel nacional no dispone de documentos que sirvan como referencia para adaptarlos a nivel local.

\section{Sostenibilidad económica}

Desde el nivel nacional se fortalecieron los procedimientos de planificación y ejecución presupuestal municipal (figura 2c). No obstante, la normativa para la inversión se orientó hacia la atención de prioridades nacionales y no contempló presupuesto para la evaluación. Acorde con la normativa, los cuatro municipios realizaron inversiones progresivas en salud, agua y saneamiento; pero enfrentaron demoras para su ejecución. Los municipios A y B destinaron parte de su

presupuesto municipal en la capacitación del personal para la EMS; mientras que los municipios C y D lo hicieron con fondos de cooperación internacional. Un problema identificado para ejercer el presupuesto fue la escasa participación de otros actores locales.

"...cuando intervienen otras instancias de gobierno, ellos no terminan de dar el fondo comprometido, lo dan hasta el siguiente año, pero ya no lo dan completo". EM-B

Según el análisis presupuestal de los años 2008, 2012, 2016 y 2018, el municipio A destinó 79\% de su presupuesto programado (124 703425 quetzales, equivalentes a 16132397 dólares estadounidenses [USD]) en salud, agua y saneamiento; el municipio B destinó 74\% (82 176895 quetzales, equivalentes a 10630905 USD); el municipio D asignó 44\% del presupuesto (16 012917 quetzales, equivalentes a 2071529 USD), y el municipio C destinó 135\% de su presupuesto (7 845305 quetzales, equivalentes a 1014917 USD). Según la normativa, todos los municipios ejercieron su presupuesto de manera adecuada, ya que está establecido ejercer como mínimo 10\% del presupuesto cada año. 


\section{Sostenibilidad social}

Los municipios con mayores avances en la dimensión social fueron los municipios B y C (figura 2d). En mecanismos de involucramiento activo de la población, se ha impulsado la participación de los Consejos de Desarrollo integrados por autoridades municipales, instituciones de gobierno y organizaciones locales.

"...cuando queremos hacer un proyecto a favor de la comunidad, hacemos enlace con los COCODES [Comités Comunales de Desarrollo], este convoca a toda la gente de las colonias en donde se intervendrá; la gente es muy colaboradora". AM-B.

En cuanto a la aceptabilidad de la EMS, la población se resiste a la implementación de proyectos cuando éstos se planifican sin su opinión. En proyectos en los que participa la comunidad en el diagnóstico y la planeación se aceptan mejor y tienen mayor demanda de continuidad.

En relación con la autonomía y capacidad propositiva de la población, en los municipios A y B se impulsaron proyectos en PS con participación de líderes comunitarios. En el municipio C, las acciones de PS se impulsaron por organismos de cooperación internacional que luego fueron adoptadas por el gobierno municipal y los consejos de desarrollo. En el municipio D estos proyectos aún dependen de organismos de cooperación internacional.

“...hay ONG [organizaciones no gubernamentales] como Save the Children. Ellos donan materiales y recursos para hacer el proyecto, nosotros aportamos una parte. Luego explicamos a la gente cómo quedaría el proyecto al final...". DPM-A.

Con respecto a la utilización de tecnologías de innovación e interacción social, se usa la plataforma de la Asamblea de Consejos de Desarrollo para difundir las acciones implementadas en comunidades. Sin embargo, la participación de líderes comunitarios en estos espacios no garantiza la comunicación efectiva a la población y autoridades municipales.

La evaluación de las dimensiones de la sostenibilidad en los cuatro municipios estudiados se presenta en la figura 3. El municipio con mayor nivel de sostenibilidad es el municipio C. En promedio se observaron puntajes de 2 en las dimensiones operativa, social y económica; la dimensión política legal alcanzó 2,5 puntos. La sostenibilidad operativa se ve afectada en forma negativa por el deficiente proceso de monitoreo y evaluación. La dimensión social se enfrenta al limitado uso de tecnologías de innovación e interacción social y la aceptabilidad de intervenciones por la población. En la dimensión económica, los municipios han mejorado la programación y ejecución de presupuesto en salud, agua y saneamiento; pero enfrentan problemas presupuestarios para financiar la evaluación. En la dimensión política legal, se identifican avances en la rendición de cuentas, planificación local y acuerdos o convenios locales; pero con vacíos en la normativa para implementación de la EMS.

\section{DISCUSIÓN}

Es evidente la ausencia de estudios previos para evaluar la sostenibilidad de intervenciones. Este es un primer estudio
FIGURA 3. Medición de las cuatro dimensiones de la sostenibilidad de la estrategia Municipios Saludables, Guatemala (2019)

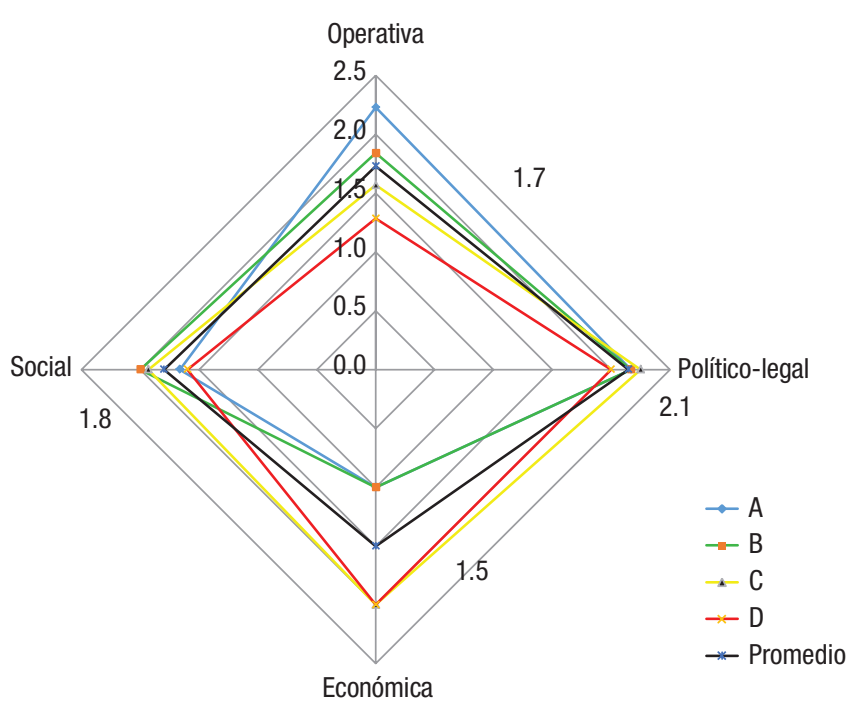

que evalúa la sostenibilidad de la EMS en Guatemala, lo cual se constituye en evidencia empírica y metodológica por las dimensiones que propone para su evaluación.

Un hallazgo relevante en la dimensión operativa de la sostenibilidad de la EMS es la disponibilidad de personal en el municipio A, que es el municipio con mayor sostenibilidad operativa. Está documentado que disponer de personal capacitado mejora la gestión e implementación de una intervención (17). Por el contrario, los municipios C y D enfrentan insuficiencia de personal para implementar la EMS. Otros países que implementaron la estrategia han enfrentado deficiencia de recursos humanos con competencias en PS. Por este motivo, disponer de personal suficiente y con competencias adecuadas es un desafío (18), pues el recurso humano es fundamental para implementar cualquier intervención $(19,20)$. En el municipio D, se observó que las nuevas autoridades no dieron continuidad a las actividades de la EMS; el cambio de gobierno y de autoridades ha sido documentado como un factor que limita la sostenibilidad operativa (9). Los avances incipientes observados en el monitoreo y la evaluación de la EMS evidencian la escasa prioridad que se le da a la evaluación, relacionada, a su vez, con una deficiente gestión. Esta situación contrasta con Argentina, donde las decisiones de la EMS se basan en información procedente de la evaluación (21).

La dimensión política legal de la sostenibilidad alcanzó el mayor puntaje, y se destacan los avances en rendición de cuentas. El menor avance se observó en el desarrollo de lineamientos nacionales y municipales para la implementación de la EMS. Un estudio que analizó la implementación y la sostenibilidad de una estrategia de salud, reconoció que contar con lineamientos claros y congruentes son una fortaleza para su aplicación (22). Pero si el marco normativo no responde al contexto, el reto de la implementación es mayor, por lo que es necesario que la normatividad se elabore en forma conjunta con representantes de la sociedad civil y otros involucrados (23).

La sostenibilidad económica alcanzó el menor avance. Abundaron los recursos financieros para agua y saneamiento; sin 
embargo, los recursos para proyectos en otras áreas de PS fueron insuficientes. Esto pone en tela de juicio el abordaje de los determinantes sociales de la salud y hace necesario convocar a actores locales para financiar proyectos más allá de agua y saneamiento. Los municipios tuvieron una inversión superior a $10 \%$ en salud, que los califica como municipios con inversión adecuada. Este dato contrasta con una investigación chilena que muestra que la inversión es menor en municipios con mayor pobreza (24), y que no es suficiente para atender su problemática de salud. Por este motivo, en este tipo de iniciativas es relevante la participación de fondos de la cooperación internacional (25).

En lo referente a dimensión social de la sostenibilidad, no se observaron grandes avances, que se explica en parte por la ausencia de mecanismos efectivos para la participación de los actores en el diseño, la implementación, la evaluación y el monitoreo de la EMS (26). Se reconoce que, para actuar sobre los determinantes sociales de la salud, los gobiernos deben abrir espacios para motivar la participación de los ciudadanos (27) y de sus organizaciones sociales (28). También se reconoce que los planes municipales que inciden en los determinantes sociales de la salud se ven fortalecidos con la participación social (29) y favorecen la continuidad de las acciones (30). Por el contrario, la escasa participación social limita la aceptabilidad de la EMS por la población (22).

Como limitaciones del estudio se pueden identificar la falta de estudios previos para evaluar la sostenibilidad de intervenciones que hubiera permitido realizar la evaluación en un período más corto. No se pudo constatar los testimonios de los informantes para las dimensiones operativa y social porque en los municipios los datos disponibles concentran, en su mayoría, información financiera.

Por último, la sostenibilidad de la EMS alcanzó un nivel regular en los municipios estudiados en Guatemala. En la dimensión operativa, se requiere mejorar la disponibilidad de personal operativo, el monitoreo y la evaluación. En la dimensión políticolegal es clara la necesidad de definir lineamientos para su implementación. En la dimensión económica, se requiere mejorar la concertación local para captar mayores recursos y financiar proyectos que incidan en los determinantes sociales de la salud. En la dimensión social, es indispensable implementar estrategias para facilitar la participación social y comunitaria de la población.

Contribución de los autores. JL concibió el estudio original, recolectó y analizó los datos, interpretó los resultados y escribió el manuscrito. RG concibió el estudio original, recolectó y analizó los datos, interpretó los resultados y escribió el manuscrito. JT recolectó los datos, interpretó los resultados y revisó el manuscrito. AS y RP interpretaron los resultados y revisaron el manuscrito. JA concibió el estudio original, analizó datos, interpretó los resultados y escribió el manuscrito. Todos los autores han leído y aprobado la versión final del manuscrito.

Agradecimientos. los autores agradecen al equipo de Promoción de la Salud del Ministerio de Salud Pública y Asistencia Social de Guatemala, que apoyó esta iniciativa desde el comienzo de la propuesta y participó en la recolección de la información.

Financiamiento. este estudio fue financiado por el Departamento de Evidencia e Inteligencia para la Acción de Salud (EIH) de la Organización Panamericana de la Salud/Organización Mundial de la Salud (OPS/OMS), la Alianza para la Investigación en Políticas y Sistemas de Salud (AHPSR) a través de la subvención "Incorporación de la investigación para avanzar en el cumplimiento de los Objetivos de Desarrollo Sostenible (ER-SDG)".

\section{Conflicto de intereses. Ninguno declarado por los autores.}

Declaración. Los patrocinadores no participaron de ninguna manera en el diseño del estudio, la colecta y análisis de los datos, la decisión de publicar este trabajo ni la preparación del manuscrito. Las opiniones expresadas en este manuscrito son responsabilidad del autor y no reflejan necesariamente los criterios ni la política de la RPSP/PAJPH y / o de la OPS.

\section{REFERENCIAS}

1. Organización mundial de la Salud. La Carta de Ottawa para la promoción de la salud. Ottawa: OMS; 1986. Disponible en: https:// www.paho.org/hq/dmdocuments/2013/Carta-de-ottawa-para-laapromocion-de-la-salud-1986-SP.pdf

2. Restrepo H. Municipios saludables: lecciones aprendidas, desafíos. Ponencia. Jornadas de Municipios saludable. Jornadas de Municipios Saludables. Buenos Aires; 2004. Disponible en: https:/ / parquedelavida.co/images/contenidos/el_parque/banco_de_conocimiento/ municipios_saludables_lecciones_aprendidas_desafios.pdf

3. Organización Panamericana de la Salud. Acerca de municipios saludables. Washington D.C.: OPS; 2020. Disponible en: https://www. paho.org/hq/index.php?option=com_content\&view=article\&i$\mathrm{d}=10706$ :about-healthy-municipalities\&Itemid=820\&lang=es

4. Alessandro L, Munist M. Municipios saludables: una opción de política pública: avances de un proceso en Argentina. Buenos Aires: OPS; 2002. Disponible en: http:/ / publicaciones.ops.org.ar/publicaciones/pubOPS_ARG/pub55.pdf

5. Eslava-Castañeda JC. Repensando la promoción de la salud en el sistema general de seguridad social en salud. Rev Salud Publica (Colombia). 2006;8(S2):106-15.
6. Reunión Centroamericana de Municipios Saludables, Ciudad Antigua, Guatemala, 28-31 de octubre, 1997. Disponible en: https:// docplayer.es/56342289-Municipios-saludables.html

7. Ministerio de Salud Pública y Acción Social de Guatemala. Guía operativa: estrategias de promoción y educación en salud, 2018 Guatemala; MSPAS; 2018. Disponible en: https:/ / pesquisa.bvsalud. org/portal/resource/pt/biblio-1024730

8. Real Academia Española. sostenible | Definición | Diccionario de la lengua española | RAE - ASALE. Disponible en: https://dle.rae.es / sostenible Acceso el 2 de febrero de 2021.

9. Buritica Marin ED, Ordoñez Mora LT. Modelo de gestión sostenible en la atención comunitaria del adulto mayor. Rev Cuba Investig Biomédicas. 2020;39(2). Disponible en: http:/ / www.revibiomedica. sld.cu/index.php/ibi/article/view/392

10. Mandl J, Málaga H. Nuevas prácticas y significados en políticas públicas saludables en los ámbitos locales de Venezuela. Rev Estud Políticas Públicas. 2019;5(1):50. Disponible en: http:/ /dx.doi. org / 10.5354/0719-6296.2019.52398

11. Hevia F, Garcia S, Cervantes M. Las organizaciones de la sociedad civil y su relación con la sociedad política y con el gobierno 
federal en México. En: IX Congreso Anual de Investigaciones sobre el Tercer Sector y VII Conferencia Regional de América Latina y el Caribe de la Sociedad Internacional de Investigación sobre el Tercer Sector, 2009. Disponible en: https://controlatugobierno. com/archivos/bibliografia/Hevia-y-Garcia-osc-y-relacion-sociedad-politica.pdf

12. Moyano-Santiago MA, Rivera-Lirio JM. El enfoque de sostenibilidad en los planes de salud de las comunidades autónomas: el desarrollo sostenible como oportunidad. Gac Sanit. 2016;30(3):1727. Disponible en: https://dialnet.unirioja.es/servlet/articulo? codigo $=5449563$

13. Sánchez Pérez MC. Satisfacción de usuarios y profesionales en la evaluación de programas sociales. Rev Evaluación Programas y Políticas Públicas. 2016;1(7):116. Disponible en: http://revistas. uned.es/index.php/REPPP/article/view/16352

14. Baca-Tavira N, Herrera Tapia F. Proyectos sociales: notas sobre su diseño y gestión en territorios rurales. Convergencia. 2016;23(72): 69-87. Disponible en: https://dialnet.unirioja.es/servlet/articulo ?codigo $=5968839$

15. Di Marco MH. ¿Quién decide qué necesitamos? Participación social y toma de decisiones en una política de abordaje territorial en Argentina. Rev Uruguaya Cienc Política. 20171;26(2).

16. Hernández Sampieri R, Fernández Collado C, Baptista Lucio P. Los procesos de la investigacion mixta. En: Metodología de la Investigación. 2014:531-86. Disponible en: https://www.uca.ac.cr/ wp-content/uploads/2017/10/Investigacion.pdf

17. Sanabria Ramos G, Pupo Ávila NL, Pérez Parra S, Ochoa Soto R, Carvajal Rodríguez C, Fernández Nieves Y. Seis décadas de avances y retos para la promoción de la salud en Cuba. Rev Cuba Salud Publica. 2018;44(3). Disponible en: http://scielo.sld.cu/scielo. php?script=sci_arttext\&pid=S0864-34662018000300003

18. Alcalde-Rabanal JE, Nigenda G, Bärnighausen T, Velasco-Mondragón HE, Darney BG. The gap in human resources to deliver the guaranteed package of prevention and health promotion services at urban and rural primary care facilities in Mexico. Hum Resour Health. 2017;15(1):48. doi: 10.1186/s12960-017-0220-5

19. Giraldo Osorio A, Velez Álvarez C. Desafíos en la formación del talento humano para atención primaria de salud en América Latina. Rev Costarric Salud Publica. 2016;25(2):54-70. Disponible en: https:/ / www.scielo.sa.cr/scielo.php?script=sci_arttext\&pid=S140914292016000200054

20. Regan S, Wong ST, Watson DE. Public perspectives on health human resources in primary healthcare: context, choices and change. Healthc Policy. 2010;5(3):e162-72. Disponible en: http://www.ncbi.nlm. nih.gov/pubmed/21286262

21. García, B; Mangiaterra VR. Sala de situación de salud local: una herramienta para el fortalecimiento de gestión de municipios y comunidades saludables. Rev Argent Salud Publica. 2018;9(34):40-3. Disponible en: http://www.scielo.org.ar/scielo.php?pid=S1853-8 10X2018000100007\&script=sci_arttext\&tlng=es
22. Montaño Arango O, Corona Armenta JR, Navarro Gómez HI, Palma Quiroz I, Rivera Gómez H, Montufar Benítez MA. Metodología para analizar las variables que impactan en la viabilidad de un proyecto para generar energía a partir de un SDF. Estudio de caso: Pachuca, Hidalgo. Acta Univ. 2019;29:1-22. Disponible en: http:// doi.org/10.15174.au.2019.2041

23. Pérez Díaz N, Suero Gutiérrez L, Veliz Gutiérrez JÁ, Linares Guerra EM, Pérez Rodríguez E. Acciones estratégicas de educación ambiental en la comunidad La Majagua para su desarrollo local. Rev Coop y Desarr. 2019;7(3):406-19. Disponible en: http://scielo.sld.cu/scielo. php?script=sci_arttext\&pid=S2310-340X2019000300406\&lng=es\& nrm=iso\&tlng=es

24. Riquelme Briceño C, Haase Delgado J, Lavanderos Bunout S, Morales Martínez A. Inequality of financial resources for primary health care. RevMedChil.2017;145(6):723-33.Disponibleen:https:/ / scielo.conicyt. cl/scielo.php?script=sci_arttext\&pid=S0034-98872017000600723\& lng=es\&nrm=iso\&tlng=es

25. Montoya CA. La formación política y participación en las organizaciones sociales en búsqueda del desarrollo comunitario. Corporación Universitaria Minuto de Dios; 2017. Disponible en: https:/ / repository.uniminuto.edu/handle/10656/10055

26. Marzán Delis M, Bonal Ruiz R, Barnett Carrion S. II Encuentro de Alcaldes y Alcaldesas de Municipios y Comunidades Saludables en la Región de las Américas. MEDISAN. 2019;23(2):394-402. Disponible en: http://scielo.sld.cu/scielo.php?script=sci_arttext\&pid $=$ S1029-30192019000200394\&lng $=$ es.

27. Lai B, Kim Y, Wilroy J, Bickel CS, Rimmer JH, Motl RW. Sustainability of exercise intervention outcomes among people with disabilities: a secondary review. 2019;41:1584-95. Disponible en: https:/ / pubmed. ncbi.nlm.nih.gov/29409367/

28. Castañeda Gamboa GI, Delgado G ME. Barreras, oportunidad y tácaticas para participar en salud según asociaciones de usuarios del valle del Cauca, Colombia. Hacia la promoción la salud. 2015;20(2):59-76.

29. Alvarado Vazquez S, Casiano Flores C. The planning conditions of public spaces in Mexico. 2020 Nov [cited 2021 Feb 3]. Disponible en: https://hal.archives-ouvertes.fr/hal-03115184

30. Picado Herrera LI. Resultados del estudio de percepciones de actores sociales sobre salud, promoción de la salud, participación social, y de planes municipales región central de occidente, Costa Rica; 2011. Univ y Salud [Internet]. 2014;1:1-17. Disponible en: http:// www.scielo.org.co/pdf/reus/v16n2/v16n2a03.pdf

Manuscrito recibido el 16 de noviembre de 2020. Aceptado para su publicación, tras revisión, el 9 de febrero de 2021. 


\section{Sustainability of the Healthy Municipalities strategy in Guatemala}

ABSTRACT Objective. Evaluate the sustainability of the Healthy Municipalities strategy in Guatemala in order to have solid evidence to support decision-making.

Methods. A concurrent mixed-methods study was carried out in five phases: 1) theoretical-conceptual (based on a narrative review of the literature on sustainability, dimensions and categories were proposed for evaluation); (2) empirical (four municipalities were selected for convenience and 29 semi-structured interviews and four focus groups were conducted with key actors to explore sustainability; with this information, a score was assigned to each category and dimension); (3) analytical, by category and dimension (content analysis was performed for qualitative information, and totals and averages were calculated for quantitative information); (4) integrative (qualitative data were integrated into matrices by category and dimension, and quantitative data were supported by qualitative information); and 5) meta-inference (consideration was given to the context and its influence on the results).

Results. Ninety-two (92) informants participated. In operational terms, progress was observed in the transfer and use of results, and in rotations in leadership. In the legal and political sphere, accountability and local planning were highlighted. In the economic sphere, progressive investment in health, water and sanitation was emphasized, as well as insufficient investment in social determinants of health. In the social sphere, few mechanisms were observed to promote and strengthen social participation.

Conclusions. In the municipalities that participated in the study, a fair level of sustainability was observed in the Healthy Municipalities strategy.

Keywords $\quad$ Evaluation study; healthy city; health promotion; local government; Guatemala.

\section{Sustentabilidade da estratégia de Municípios Saudáveis na Guatemala}

RESUMO Objetivo. Avaliar a sustentabilidade da estratégia de Municípios Saudáveis na Guatemala para dispor de evidências sólidas para apoiar o processo decisório.

Métodos. Foi realizado um estudo de método misto concorrente em cinco fases: 1) fase teórica-conceitual em que foi feita a revisão narrativa da literatura em sustentabilidade a partir da qual foram propostas dimensões e categorias a serem avaliadas; 2) fase empírica em que foi feita a seleção por conveniência de quatro municípios, com 29 entrevistas semiestruturadas e quatro grupos de discussão com as principais partes interessadas para explorar a sustentabilidade; a partir das informações coletadas, foi dada uma pontuação a cada categoria e dimensão; 3) fase analítica, por categoria e dimensão, em que foi realizada a análise do conteúdo para os dados qualitativos e feito o cálculo de somatórias e médias para os dados quantitativos; 4) fase integrativa em que os dados qualitativos foram integrados em matrizes por categoria e dimensão e os dados quantitativos foram respaldados com a informação qualitativa e 5) metainferência em que foi analisado o contexto e sua influência nos resultados.

Resultados. O estudo incluiu 92 participantes. Na dimensão operacional, destacam-se os avanços na transferência e no uso de resultados, bem como a ênfase em liderança. Na dimensão jurídico-política, destacam-se a prestação de contas e os planos locais. Na dimensão econômica, destaca-se o investimento progressivo em saúde, água e saneamento, com investimento inadequado nos determinantes sociais da saúde. Na dimensão social, observam-se poucos mecanismos para incentivar e reforçar a participação social.

Conclusões. Foi observado nos municípios participantes do estudo um nível constante de sustentabilidade da estratégia de Municípios Saudáveis.

Palavras-chave Estudo de avaliação; cidade saudável; promoção da saúde; governo local; Guatemala. 